\title{
BMJ Open Can exercise delay transition to active therapy in men with low-grade prostate cancer? A multicentre randomised controlled trial
}

\author{
Daniel A Galvão, ${ }^{1,2}$ Dickon Hayne, ${ }^{3,4}$ Mark Frydenberg, ${ }^{5}$ Suzanne K Chambers, ${ }^{1,6,7,8}$ \\ Dennis R Taaffe, ${ }^{1,2,9}$ Nigel Spry, ${ }^{1,10,11}$ Paul A Scuffham, ${ }^{6,12}$ Robert S Ware, ${ }^{6}$ \\ Nicolas H Hart, ${ }^{1,2}$ Robert U Newton ${ }^{1,2,13}$
}

To cite: Galvão DA, Hayne D, Frydenberg $\mathrm{M}$, et al. Can exercise delay transition to active therapy in men with low-grade prostate cancer? A multicentre randomised controlled trial. BMJ Open 2018;8:e22331. doi:10.1136/ bmjopen-2018-022331

- Prepublication history for this paper is available online. To view these files, please visit the journal online (http://dx.doi org/10.1136/bmjopen-2018022331).

Received 14 February 2018 Revised 7 March 2018 Accepted 8 March 2018

Check for updates

For numbered affiliations see end of article.

Correspondence to Professor Daniel A Galvão; d.galvao@ecu.edu.au

\section{ABSTRACT}

Introduction Active surveillance is a strategy for managing low-risk, localised prostate cancer, where men are observed with serial prostate-specific antigen assessments to identify signs of disease progression. Currently, there are no strategies to support active surveillance compliance nor are there interventions that can prevent or slow disease progression, ultimately delaying transition to active treatment before it is clinically required. Recently, we proposed that exercise may have a therapeutic potential in delaying the need for active treatment in men on active surveillance.

Methods and analysis A single-blinded, two arm, multicentre randomised controlled trial will be undertaken with 168 patients randomly allocated in a ratio of 1:1 to exercise or usual care. Exercise will consist of supervised resistance and aerobic exercise performed three times per week for the first 6 months in an exercise clinical setting, and during months 7-12, a progressive stepped down approach will be used with men transitioning to once a week supervised training. Thereafter, for months 13 to 36 , the men will self-manage their exercise programme. The primary endpoint will be the time until the patients begin active therapy. Secondary endpoints include disease progression (prostate specific antigen), body composition and muscle density, quality of life, distress and anxiety and an economic analysis will be performed. Measurements will be undertaken at 6 and 12 months (postintervention) and at 24 and 36 months follow-up. The primary outcome (time to initiation of curative therapy) will be analysed using Cox proportional hazards regression. Outcomes measured repeatedly will be analysed using mixed effects models to examine between-group differences. Data will be analysed using an intention-to-treat approach. Ethics and dissemination Outcomes from the study will be published in peer-reviewed academic journals and presented in scientific, consumer and clinical meetings. Trial registration number ACTRN12618000225213.

\section{INTRODUCTION}

Prostate cancer (PCa) is a major challenge for our health system and its workforce and for Australian society as a whole. In 2017,
Strengths and limitations of this study

- This is a novel multicentre randomised controlled trial of 12-month supervised resistance and aerobic exercise versus usual care in men with prostate cancer on active surveillance, with subsequent follow-up of over 2 years to monitor therapeutic progression and psychological well-being.

- The study proposed here will determine the efficacy of a comprehensive exercise programme during active surveillance in delaying or preventing patient morbidity associated with prostate cancer primary therapy. Savings to the health and medical system could be extensive with any delay in prostatectomy, radiation therapy or androgen deprivation therapy, reducing costs of current overtreatment substantially.

- The study will be undertaken in the Australian clinical setting of prostate cancer care, and differences in active surveillance and disease progression definitions may exist within the context of other countries.

more than 200000 Australian men were living with $\mathrm{PCa}, 80 \%$ of them were longterm survivors. ${ }^{1} 2$ The widespread prostate-specific antigen (PSA) screening for PCa has led to concerns about the overdiagnosis and overtreatment of this disease with the overdiagnosis estimated to be as high as $67 \% .^{3}$ Overtreatment rates are also high with reports of $80 \%$ of men receiving treatments on initial diagnosis. ${ }^{4}$ Active surveillance (AS) is a strategy for managing low-risk localised PCa, where men are observed with regularly schedule serial PSA assessments to identify signs of disease progression. ${ }^{5}$ AS reduces overtreatment in clinically insignificant disease, ${ }^{5}$ reducing $\mathrm{PCa}$ burden for the individual, the healthcare system and the society. Problematically, most men will not comply with AS and those who convert to active treatment too 


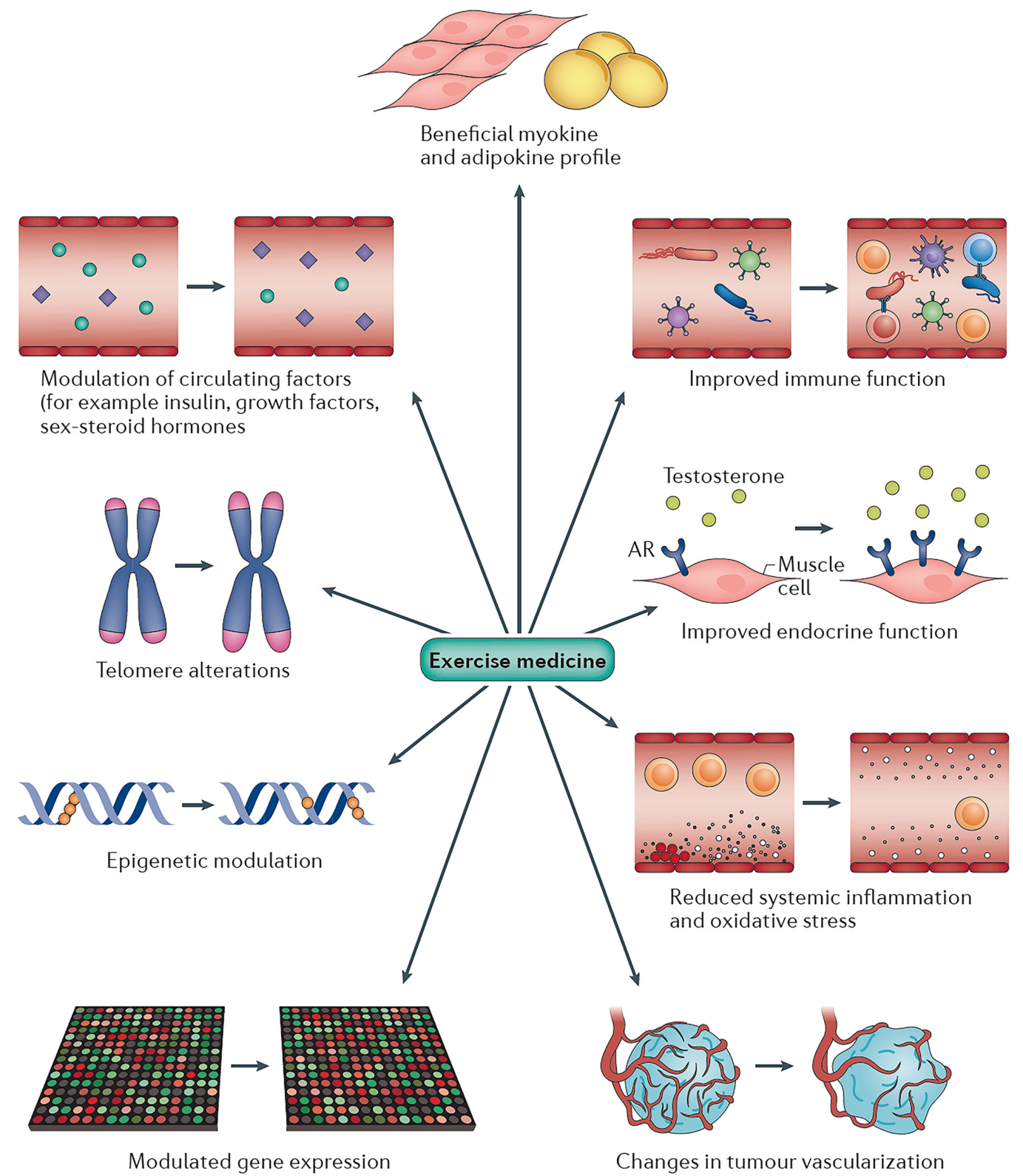

Figure 1 Proposed mechanisms of exercise on prostate cancer and disease progression. Reprinted with permission from Galvao et al. ${ }^{6}$

soon. Currently there are no strategies to (1) support AS compliance applying a lifestyle approach or (2) prevent or slow disease progression, ultimately delaying transition to active treatment before it is clinically required..$^{5-10}$ These are the two critical unanswered questions in applying AS to men with PCa.

We recently presented a preliminary evidence ${ }^{6}$ that exercise may have therapeutic potential in delaying the need for active treatment (eg, surgery/radiation) in men on AS. Exercise is implicated in decreasing the number of patients undergoing active treatment, ${ }^{11}$ reducing PSA, ${ }^{12}$ as well as modulating the biological processes involved with tumourigenesis. ${ }^{13}$ It may act through systemic mechanisms, as exercise alters circulating factors that inhibit PCa cell proliferation in vitro. ${ }^{14-16}$ Further, increased adiposity is associated with increased risk of $\mathrm{PCa}$ progression specifically during $\mathrm{AS},{ }^{17}$ and exercise can be an effective countermeasure to this in the setting of AS (figure 1). ${ }^{6}$

Exercise as a therapeutic intervention in such men may also have important additional benefits by addressing other adverse consequences. The psychological burden of AS, in terms of cancer-related anxiety, also influences adherence and treatment decisions as much as clinical disease progression. ${ }^{18}$ Not treating a cancer runs against the norm and the surveillance process is inherently stressful. Exercise presents as a supportive care approach that closely maps onto core male values and has the potential to both combat illness but also help prevent and/or minimise anxiety and uncertainty related to living with a PCa diagnosis. Specifically, by supporting male values around strength, self-reliance and action, ${ }^{19}$ exercise may 


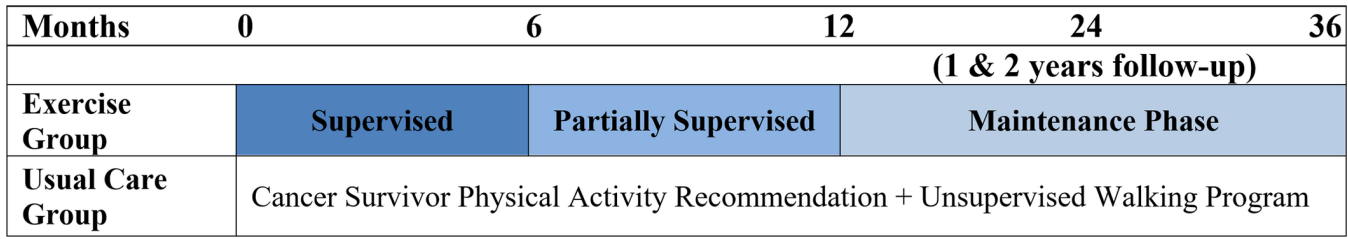

Figure 2 Study design (1 year intervention and 1 and 2 years follow-up).

increase men's self-efficacy or feelings of control over their health, and thereby support adherence to AS while improving psychological outcomes and physical and mental quality of life (QoL). ${ }^{60}$

To characterise and quantify the benefits of exercise for men on AS, we propose to undertake a multicentre randomised controlled trial (RCT) of 12-month supervised resistance and aerobic exercise versus usual care in men with PCa on AS, with subsequent follow-up of over 2 years to monitor therapeutic progression and psychological well-being.

\section{METHODS AND ANALYSIS}

This is a single-blinded (investigators blinded to group allocation), two-arm, multicentre RCT that will examine the efficacy of combined resistance and aerobic exercise during AS on disease progression in men with low-risk PCa. An 'exercise' group will complete a 6-month supervised exercise intervention followed by a stepped down programme for 6 months and a subsequent self-managed maintenance programme for 2 years (figure 2). A 'usual care' group will maintain usual medical care and receive standard information on current physical activity guidelines for cancer survivors, which will include an unsupervised walking programme.

\section{Patients and methods}

One hundred and sixty-eight men (84 patients per arm) within 1 year of diagnosis of $\mathrm{PCa}$ and undergoing AS will be recruited by invitation of their attending specialist from two Australian cities (Perth and Melbourne). Patients suitable for AS will be selected from the Multi-Disciplinary Uro-Oncology meeting at the recruiting site. Inclusion criteria are: (1) histologically proven adenocarcinoma of the prostate, (2) no prior therapy for $\mathrm{PCa}$, (3) fit for curative intent therapy, (4) willing to attend follow-up, (5) clinical stage $\leq \mathrm{T} 2,(6)<10 \%$ Gleason pattern 4 disease on biopsy and (7) PSA $\leq 10 \mathrm{ng} / \mathrm{mL}$. Exclusion criteria are: (1) already performing regular exercise defined as undertaking structured resistance and aerobic training two or more times per week within the past 3 months, (2) acute illness or any musculoskeletal, cardiovascular or neurological disorder that could inhibit exercise performance or put participants at risk from exercising, (3) variant histopathology (small cell, intraductal, sarcomatoid), presence of extraprostatic extension or lymphovascular invasion, (4) patient no longer considered a candidate for curative intent treatment and (5) intention to move place of residence away from the two study sites. Eligible patients will undertake baseline measurements prior to randomisation. All patients must provide written informed consent prior to participation in addition to a physician consent form. The study coordinator will obtain the consent forms from patients and physicians. All data relevant to the study will be kept on password-encrypted computers accessible only by study investigators situated in the Exercise Medicine Research Institute (Perth, WA, Australia).

\section{Patient and public involvement}

We work closely with the Prostate Cancer Foundation of Australia (PCFA), their support groups and state offices. PCFA and support groups have been involved in the development of this proposal and will maintain a very important role on this project in patient recruitment and support as well as translation and dissemination of the research findings. We have used this feedback to inform this project and ensure that it engages participants in a respectful, ethical and impactful way. As the project evolves, PCFA will assist in the dissemination of findings to cancer support groups and the general public, while study participants will receive their individual results as well as overall study findings.

\section{Randomisation}

Patients will be randomly allocated in a ratio of $1: 1$ to the two study arms, and will be stratified according to: (1) age $(<60$ years $\geq)$, (2) PSA $(<5 \geq)$ and (3) time on AS $(<6$ months $\geq$ ). Within each stratum, randomisation will occur in blocks of either 8 or 10, with block size randomly selected in a 1:1 ratio. Patients will be randomised via a central web-based service (Griffith University) to ensure allocation concealment until study entry. Randomisation will occur immediately after each patient completes their baseline questionnaire (figure 3 ). The study coordinator will assign participants to groups.

\section{Measurements}

All measurement study endpoints will take place at baseline, 6 months, postintervention (12 months) and 1 and 2-year follow-up (months 24 and 36). All assessment tools/procedures have established validity and reliability and are used widely in clinical research including by our team.

\section{Primary study endpoint}

Patients undergoing active therapy

The number of patients undergoing active therapy (radical prostatectomy, radiotherapy or androgen deprivation) and the time they began active therapy will be extracted directly from the urologist clinical investigators 


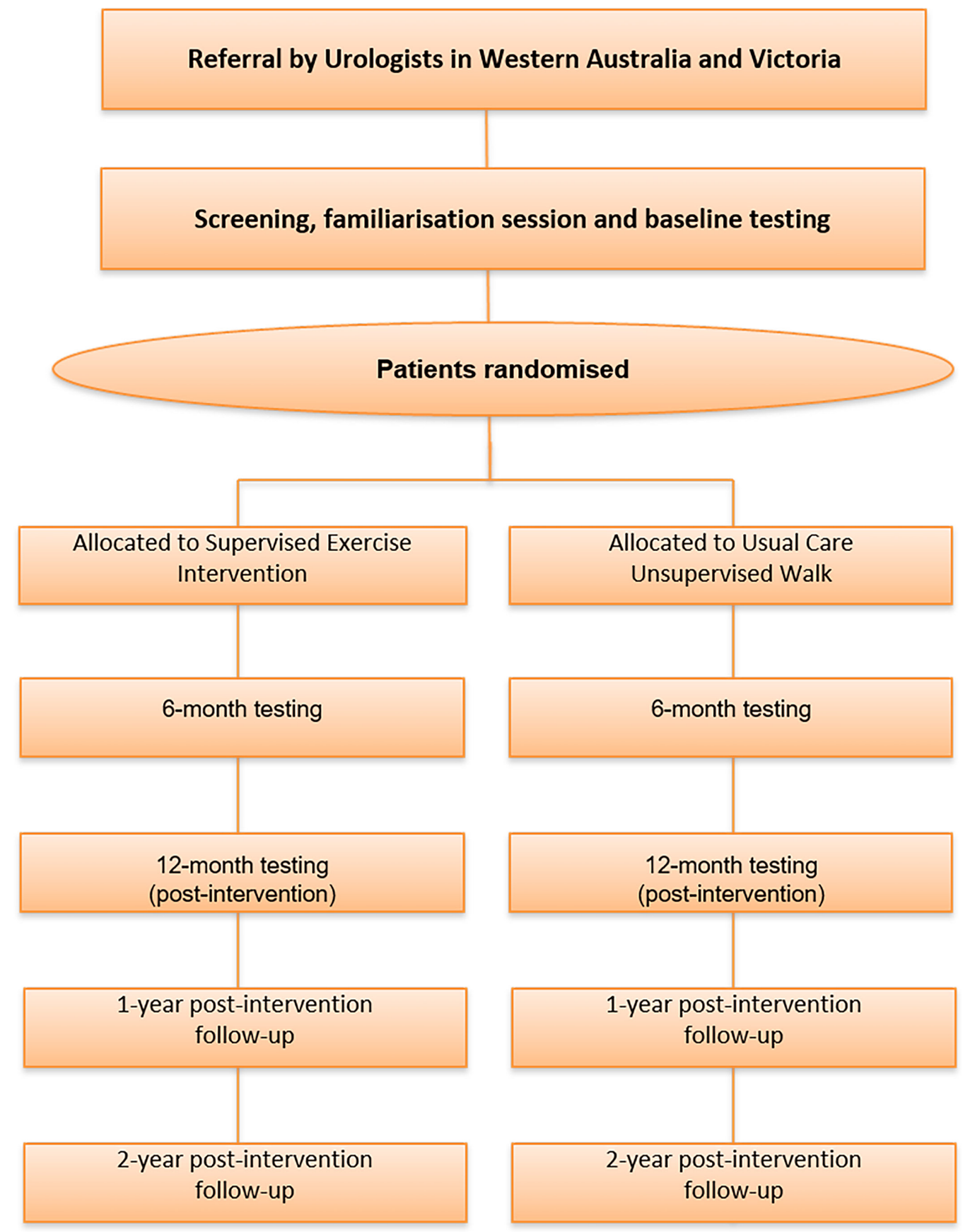

Figure 3 CONSORT diagram. CONSORT, Consolidated Standards of Reporting Trials.

as part of their routine clinical practice; therefore, all patients will be captured. Data will be extracted for the 3 years following randomisation, and the reasons for progression to active therapy will be recorded.

\section{Secondary study endpoints}

Disease progression (PSA)

For all patients, PSA will be measured commercially by an Australian National Association of Testing Authorities accredited laboratory. ${ }^{21}$

Body composition and muscle density

Regional and whole-body lean mass and fat mass will be derived from the whole-body dual-energy X-ray absorptiometry scans. Trunk adiposity, visceral fat and adipose indices will be assessed using standard procedures. $^{21-23}$ Peripheral quantitative CT will be used to measure muscle density (an indicator of fat infiltration within the muscle) and muscle cross-sectional areas of the lower limbs. ${ }^{24}$

QoL, prostate cancer-specific distress, overall psychological distress and PSA anxiety

Health-related QoL will be assessed using the Medical Outcomes Short Form 36 (SF-36v2).$^{25}$ A three-item cancer standardised anxiety scale developed by Latini $e a^{18}$ will assess men's fears about cancer recurrence. This scale has 
previously been found to predict receipt of active treatment independent of PSA velocity in a sample of men on active surveillance. ${ }^{18}$ Overall cancer-specific distress will be assessed by the Impact of Events Scale revised ${ }^{26}$ which has been used extensively by our team and is valid and reliable in patients with prostate cancer. ${ }^{27} 28$ The Brief Symptom Inventory-18 will be used to assess psychological distress across the following domains: anxiety, depression, somatisation and global distress severity. ${ }^{29}$ Antidepressant use will be recorded. The Memorial Anxiety Scale for Prostate Cancer will be used to assess anxiety specifically focused on PSA testing (the PSA Anxiety Subscale). ${ }^{30}$

\section{Economic analysis}

An alongside trial economic evaluation will be undertaken to assess the health benefits and additional costs compared with usual care. This will inform the relative value for money of exercise compared with other healthcare interventions in this group of men with PCa. Hospital resource use and associated costs will be obtained to assess costs for secondary healthcare for the exercise and usual care groups. All hospital events, including emergency department attendances, outpatient visits and procedures and inpatient admission for all causes will be sought. These data are important to identify potential PCa-related events as well as identifying total healthcare resource use. In addition, the costs for providing the exercise intervention will be identified through the study. Data on health benefits and costs will be adjusted for covariates such as age, key comorbidities (eg, diabetes), body mass index and so forth. Health benefits will be measured as quality of life via the SF-36v2 and converted to a health utility scale using the Australian SF-6D scoring algorithm. ${ }^{31}$ Costs associated with health resource use and the costs of the intervention will be standardised to a common year (eg, 2018). Incremental costs and benefits will then be estimated and reported as the incremental cost utility ratio (ICUR). The ICUR will be bootstrapped ${ }^{32}$ to identify the $95 \%$ CIs, probability the intervention is good value for money and the risk of anyone in the intervention group not being better off. Deterministic sensitivity analysis will be undertaken to identify the main drivers of the costs, outcomes and value for money.

\section{Effect mediators}

The Masculinity in Chronic Disease Scale ${ }^{19}$ will assess the extent to which men identify with six masculine values: strength; sexual importance/priority; family responsibilities; emotional self-reliance; optimistic capacity and action approach. This scale was developed for Australian men with prostate cancer; has strong evidence of validity in this target group and has been found to predict men's help seeking. ${ }^{33}$ The General Self-Efficacy Scale $^{34}$ will assess men's perceptions that they can cope with stressful life events. This scale has been used widely internationally as a predictor of adaptation to adversity including cancer treatment effects. ${ }^{20}$
Other measurements: physical function and physical activity levels A battery of standard tests will be used to assess physical function: (1) $400 \mathrm{~m}$ walk (aerobic capacity), (2) one repetition maximum for the leg press and chest press (muscular strength) and (3) repeated chair rise (lower body muscle function). ${ }^{21-23}$ Physical activity levels will be assessed objectively over a 7-day period using a validated, reliable triaxial accelerometer activity monitor (ActiGraph GT3X+) ${ }^{35}$ Self-reported physical activity will be assessed by the leisure score index from the Godin Leisure-Time Exercise Questionnaire. ${ }^{23} 3637$

\section{Safety and monitoring}

Patients will be monitored for any adverse events during training and testing by the exercise physiologists with study clinicians overseeing all aspects of patient management where required.

\section{Exercise intervention}

The exercise intervention will consist of supervised resistance and aerobic exercise performed over three sessions per week for 6 months in an exercise clinic setting. During months 7-12, the exercise intervention group will transition to once a week supervised exercise in a clinic for months 7-8, once every 2 weeks supervised exercise in months 9-10 and once per month supervised exercise in months 11-12. This step-down approach will include a self-managed exercise programme consisting of a booklet and training log with detailed information about the exercise prescription and how to implement in a variety of settings. Participants will be encouraged and supported to continue their aerobic and resistance exercise programme in their local fitness centre or other exercise facilities where they can maintain a high-quality exercise programme under their self-management. The self-managed exercise programme is designed to replicate the exercises performed in the supervised sessions and includes resistance, aerobic and flexibility exercises. Finally, from month 13 to 36, the exercise programme will exclusively consist of a self-managed programme and has been implemented by our group previously in a multicentre year-long trial in men with PCa on active therapy. ${ }^{23}$ The initial programme will be supervised by exercise physiologists in Perth and Melbourne.

The exercise programme is designed to provide optimal stimulus to the cardiorespiratory and neuromuscular systems while maximising safety, compliance and retention. The exercise sessions will be conducted in small groups of up to 10-12 participants exercising in pairs under direct supervision to ensure correct technique and minimise the risk for injury. Resistance exercise will involve 6-8 exercises that target the major upper and lower body muscle groups. Intensity will be manipulated from 6 to 12 repetition maximum (RM; ie, the maximal weight that can be lifted 6 to 12 times which is equivalent to $\sim 60 \%-85 \%$ of $1 \mathrm{RM}$ ) using one to four sets per exercise. The aerobic exercise component will include 20 to 30 min of moderate to vigorous intensity cardiovascular 
exercise ( $\sim 60 \%-85 \%$ of estimated maximum heart rate) using a variety of modes such as walking or jogging on a treadmill, cycling or rowing on a stationary ergometer. Participants will be encouraged to undertake additional aerobic exercise outside the clinic sessions with the goal of achieving a total of at least $150 \mathrm{~min}$ of moderate to vigorous intensity aerobic exercise each week. Exercise prescription will be progressive and modified according to individual response. Both moderate intensity continuous and high-intensity interval training will be implemented to provide greater variety and training stimulus. To reduce the possibility of boredom and over-reaching, the exercise programme will be periodised by cycling emphasis on intensity and volume. We have used this exercise prescription effectively in previous trials involving men with PCa and have reported significant improvements in quality of life, lean muscle mass, fatigue, aerobic capacity and physical function. ${ }^{21} 23$ 38-48 The step-down approach to self-management is intended to maximise the translation of this intervention to best practice management of this patient population.

\section{Usual care}

The usual care group will receive an unsupervised walking programme and an educational booklet outlining the current national physical activity recommendation for cancer survivors along with a logbook to record their physical activity. During the study, both groups will be encouraged to maintain customary dietary patterns and the Mini Nutritional Assessment will be used to monitor nutritional status. ${ }^{49}$

\section{Calculation of sample size}

The target sample size of 168 patients is based on having $80 \%$ power to be able to detect a HR of undergoing curative therapy in the exercise intervention group, compared with the usual care group, of 0.35 or smaller $($ alpha $=0.05)$. This is equivalent to a difference between $25 \%$ of the usual care group undergoing curative therapy within 3 years postrandomisation versus $<10 \%$ in the group undertaking the exercise intervention. ${ }^{11} \mathrm{~A}$ difference of this magnitude has been recommended by our practice clinicians to be clinically important. This sample size accounts for a possible 5\% dropout due to clinical records not being available at study completion. This sample size will give us sufficient power to examine our secondary outcomes of interest. For example, for muscle mass we will have $>80 \%$ power to detect differences between groups of $0.8 \mathrm{~kg}$ or greater at 6 months postintervention, assuming an SD for change of $\sim 1.5 \mathrm{~kg}$ and $30 \%$ loss to follow-up (due to the need for participants to represent to the study team for this outcome to be measured). ${ }^{21}$

\section{Statistical analysis}

The primary outcome, initiation of curative therapy, will be analysed using Cox proportional hazards regression, with treatment group (exercise intervention/usual care) entered as the main effect. If data do not meet the proportional hazards assumption, the log-rank test will be used. Outcomes measured repeatedly will be analysed using mixed-effects models to examine differences between groups over time, with treatment group and time included as main effects, as well as an intervention-by-time interaction term. The patient will be included as a random effect to account for the non-independence of observations from the same participant. Continuous outcomes will be analysed using linear models, binary outcomes with logistic models and count outcomes with Poisson models. Depending on the balance between groups postrandomisation, clinically relevant covariates will be included in the models where appropriate. Data will be analysed using an intention-to-treat approach. Tests will be two tailed and an alpha level of 0.05 will be applied as the criterion for statistical significance.

\section{ETHICS AND DISSEMINATION}

Outcomes from the study will be published in peer-reviewed academic journals and presented in scientific, consumer and clinical meetings. The study investigators and trial coordinator will have access to the data.

\section{DISCUSSION}

PCa is a financial burden to men and their families and our health system. In 2008 and 2009, this was the top male cancer cost to the health system in Australia, totalling $\$$ A347 million or $14 \%$ of total male cancer expenditure. ${ }^{50}$ As PCa prevalence increases, the health system will find it increasingly difficult to prioritise care in the absence of clearly developed economic models. There is a deepening realisation by clinicians and patients that many prostate cancers do not require active treatment, and in fact, such interventions may result in devastating adverse effects. Already, a considerably large number of patients are referred for AS. ${ }^{751-53}$ Studies (including our previous work) examining the role of exercise in PCa provide strong evidence that beneficial effects are derived during or following definitive therapy and exercise has recently been included in the 2015 PCa Care Guideline: American Society of Clinical Oncology Clinical Practice Guideline. ${ }^{7}$ There is, however, no information on and evidence base for the efficacy of exercise medicine interventions during AS in slowing disease progression, reducing anxiety and distress and improving QoL. The study proposed here would be the first to determine the efficacy of a comprehensive exercise programme during AS in substantially delaying or preventing patient morbidity associated with PCa primary therapy. Savings to the health and medical system could be extensive with any delay in prostatectomy, radiation therapy or androgen deprivation therapy reducing costs of current overtreatment substantially. If proven efficacious in this stage of PCa management, exercise medicine could be immediately implemented as an important component of clinical best practice, at minimal 
cost and with no side effects. This will dramatically reduce overtreatment, saving the patient considerable suffering, physical pain and possibly extending survival.

\section{Author affiliations}

${ }^{1}$ Exercise Medicine Research Institute, Edith Cowan University, Joondalup, Western Australia, Australia

${ }^{2}$ School of Medical and Health Sciences, Edith Cowan University, Joondalup, Western Australia, Australia

${ }^{3}$ Fiona Stanley Hospital, Murdoch, Western Australia, Australia

${ }^{4}$ UWA Medical School, University of Western Australia, Perth, Western Australia,

Australia

${ }^{5}$ Department of Surgery, Monash University, Melbourne, Victoria, Australia

${ }^{6}$ Menzies Health Institute Queensland, Griffith University, Brisbane, Queensland,

Australia

${ }^{7}$ Centre for Research in Cancer Control, Cancer Council Queensland, Brisbane, Queensland, Australia

${ }^{8}$ Prostate Cancer Foundation of Australia, Sydney, New South Wales, Australia ${ }^{9}$ School of Human Movement and Nutrition Sciences, The University of Queensland, Brisbane, Queensland, Australia

${ }^{10}$ Genesis CancerCare, Joondalup, Western Australia, Australia

${ }^{11}$ Faculty of Medicine, University of Western Australia, Perth, Western Australia, Australia

${ }^{12}$ Centre for Applied Health Economics, School of Medicine, Griffith University,

Brisbane, Queensland, Australia

${ }^{13}$ UQ Centre for Clinical Research, University of Queensland, Brisbane, Queensland, Australia

Contributors DAG, DH, MF, SKC, DRT, NS and RUN developed the study concept and protocols and initiated the project. PAS, RSW and NHH assisted in further development of the protocol. DAG, DH, MF, SKC, DRT, NS, PAS, RSW, NHH and RUN drafted the manuscript. DAG, DH, MF, DRT, NHH and RUN will implement the protocol and oversee the collection of the data. All authors contributed and approved the final manuscript.

Funding This study was funded by the National Health and Medical Research Council (NHMRC) APP1147137. DAG is funded by a Cancer Council Western Australia Research Fellowship. SKC is supported by an Australian Research Council Professorial Future Fellowship. NHH is funded by a Cancer Council Western Australia Postdoctoral Research Fellowship.

Competing interests None declared.

Patient consent Not required.

Ethics approval Edith Cowan University Human Ethics Committee (ID: 17072 GALVAO).

Provenance and peer review Not commissioned; peer reviewed for ethical and funding approval prior to submission.

Open Access This is an Open Access article distributed in accordance with the Creative Commons Attribution Non Commercial (CC BY-NC 4.0) license, which permits others to distribute, remix, adapt, build upon this work non-commercially, and license their derivative works on different terms, provided the original work is properly cited and the use is non-commercial. See: http://creativecommons.org/ licenses/by-nc/4.0/

(c) Article author(s) (or their employer(s) unless otherwise stated in the text of the article) 2018. All rights reserved. No commercial use is permitted unless otherwise expressly granted.

\section{REFERENCES}

1. Yu XQ, Smith DP, Clements MS, et al. Projecting prevalence by stage of care for prostate cancer and estimating future health service needs: protocol for a modelling study. BMJ Open 2011;1:e000104.

2. Yu XQ, Luo Q, Smith DP, et al. Prostate cancer prevalence in New South Wales Australia: a population-based study. Cancer Epidemiol 2015;39:29-36.

3. Draisma G, Etzioni R, Tsodikov A, et al. Lead time and overdiagnosis in prostate-specific antigen screening: importance of methods and context. J Natl Cancer Inst 2009;101:374-83.
4. Hoffman KE, Niu J, Shen Y, et al. Physician variation in management of low-risk prostate cancer: a population-based cohort study. JAMA Intern Med 2014;174:1450-9.

5. Klotz L. Active surveillance for prostate cancer: for whom? J Clin Oncol 2005;23:8165-9.

6. Galvão DA, Taaffe DR, Spry N, et al. Enhancing active surveillance of prostate cancer: the potential of exercise medicine. Nat Rev Urol 2016;13:258-65.

7. Chen RC, Rumble RB, Loblaw DA, et al. Active surveillance for the management of localized prostate cancer (Cancer Care Ontario Guideline): american society of clinical oncology clinical practice guideline endorsement. J Clin Oncol 2016;34:2182-90.

8. Slomski A. Expert panel advocates surveillance for men with low-risk prostate cancer. JAMA 2012;307:133.

9. Cooperberg MR, Carroll PR, Klotz L. Active surveillance for prostate cancer: progress and promise. J Clin Oncol 2011;29:3669-76.

10. Thompson IM, Klotz L. Active surveillance for prostate cancer. JAMA 2010;304:2411-2.

11. Frattaroli J, Weidner G, Dnistrian AM, et al. Clinical events in prostate cancer lifestyle trial: results from two years of follow-up. Urology 2008;72:1319-23.

12. Ornish D, Weidner G, Fair WR, et al. Intensive lifestyle changes may affect the progression of prostate cancer. J Urol 2005;174:1065-70. discussion 69-70.

13. Ornish D, Magbanua MJ, Weidner G, et al. Changes in prostate gene expression in men undergoing an intensive nutrition and lifestyle intervention. Proc Natl Acad Sci U S A 2008;105:8369-74.

14. Rundqvist $\mathrm{H}$, Augsten $M$, Strömberg $A$, et al. Effect of acute exercise on prostate cancer cell growth. PLoS One 2013;8:e67579.

15. Barnard RJ, Ngo TH, Leung PS, et al. A low-fat diet and/or strenuous exercise alters the IGF axis in vivo and reduces prostate tumor cell growth in vitro. Prostate 2003;56:201-6.

16. Leung PS, Aronson WJ, Ngo TH, et al. Exercise alters the IGF axis in vivo and increases p53 protein in prostate tumor cells in vitro. $\mathrm{J} \mathrm{Appl}$ Physiol 2004;96:450-4.

17. Bhindi B, Kulkarni GS, Finelli A, et al. Obesity is associated with risk of progression for low-risk prostate cancers managed expectantly. Eur Urol 2014;66:841-8.

18. Latini DM, Hart SL, Knight SJ, et al. The relationship between anxiety and time to treatment for patients with prostate cancer on surveillance. J Urol 2007;178:826-32.

19. Chambers SK, Hyde MK, Oliffe JL, et al. Measuring masculinity in the context of chronic disease. Psychol Men Masc 2015;17:228-42.

20. Mystakidou K, Tsilika E, Parpa E, et al. Relationship of general selfefficacy with anxiety, symptom severity and quality of life in cancer patients before and after radiotherapy treatment. Psychooncology 2013;22:1089-95.

21. Galvão DA, Taaffe DR, Spry N, et al. Combined resistance and aerobic exercise program reverses muscle loss in men undergoing androgen suppression therapy for prostate cancer without bone metastases: a randomized controlled trial. J Clin Oncol 2010;28:340-7.

22. Galvão DA, Taaffe DR. Resistance exercise dosage in older adults: single- versus multiset effects on physical performance and body composition. J Am Geriatr Soc 2005;53:2090-7.

23. Galvão DA, Spry N, Denham J, et al. A multicentre year-long randomised controlled trial of exercise training targeting physical functioning in men with prostate cancer previously treated with androgen suppression and radiation from TROG 03.04 RADAR. Eur Urol 2014;65:856-64.

24. Taaffe DR, Henwood TR, Nalls MA, et al. Alterations in muscle attenuation following detraining and retraining in resistance-trained older adults. Gerontology 2009;55:217-23.

25. Ware JE, Sherbourne CD. The MOS 36-item short-form health survey (SF-36). I. Conceptual framework and item selection. Med Care 1992;30:473-83.

26. Horowitz M, Wilner N, Alvarez W. Impact of event scale: a measure of subjective stress. Psychosom Med 1979;41:209-18.

27. Chambers SK, Zajdlewicz L, Youlden DR, et al. The validity of the distress thermometer in prostate cancer populations. Psychooncology 2014;23:195-203.

28. McDowell ME, Occhipinti S, Gardiner RA, et al. Prevalence and predictors of cancer specific distress in men with a family history of prostate cancer. Psychooncology 2013;22:2496-504.

29. Zabora J, BrintzenhofeSzoc K, Jacobsen P, et al. A new psychosocial screening instrument for use with cancer patients. Psychosomatics 2001;42:241-6.

30. Roth AJ, Rosenfeld B, Kornblith $A B$, et al. The memorial anxiety scale for prostate cancer: validation of a new scale to measure anxiety in men with with prostate cancer. Cancer 2003;97:2910-8. 
31. Norman R, Viney R, Brazier J, et al. Valuing SF-6D Health States Using a Discrete Choice Experiment. Med Decis Making 2014;34:773-86.

32. Efron B. Better bootstrap confidence intervals. J Am Stat Assoc 1987;82:171-85.

33. Hyde MK, Zajdlewicz L, Wootten AC, et al. Medical help-seeking for sexual concerns in prostate cancer survivors. Sex Med 2016;4:e7-e17.

34. Schwarzer R, Jerusalem M. Generalized self-efficacy scale. In: Weinman J, Wright S, Johnston M, eds. Measures in health psychology: a user's portfolio Causal and control beliefs. UK: Windsor, 1995:35-7.

35. Plasqui G, Westerterp KR. Physical activity assessment with accelerometers: an evaluation against doubly labeled water. Obesity 2007:15:2371-9.

36. Godin G, Shephard RJ. A simple method to assess exercise behavior in the community. Can J App/ Sport Sci 1985;10:141-6.

37. Galvão DA, Newton RU, Gardiner RA, et al. Compliance to exerciseoncology guidelines in prostate cancer survivors and associations with psychological distress, unmet supportive care needs, and quality of life. Psychooncology 2015:1241-9.

38. Galvão DA, Newton RU. Review of exercise intervention studies in cancer patients. J Clin Oncol 2005;23:899-909.

39. Galvão DA, Nosaka K, Taaffe DR, et al. Endocrine and immune responses to resistance training in prostate cancer patients. Prostate Cancer Prostatic Dis 2008;11:160-5.

40. Galvão DA, Nosaka K, Taaffe DR, et al. Resistance training and reduction of treatment side effects in prostate cancer patients. Med Sci Sports Exerc 2006;38:2045-52.

41. Galvão DA, Taaffe DR, Spry N, et al. Acute versus chronic exposure to androgen suppression for prostate cancer: impact on the exercise response. J Urol 2011;186:1291-7.

42. Galvão DA, Taaffe DR, Spry N, et al. Physical activity and genitourinary cancer survivorship. Recent Results Cancer Res 2011;186:217-36.

43. Cormie P, Newton RU, Taaffe DR, et al. Exercise maintains sexual activity in men undergoing androgen suppression for prostate cancer: a randomized controlled trial. Prostate Cancer Prostatic Dis 2013;16:170-5.

44. Cormie P, Newton RU, Spry N, et al. Safety and efficacy of resistance exercise in prostate cancer patients with bone metastases. Prostate Cancer Prostatic Dis 2013;16:328-35.

45. Beydoun N, Bucci JA, Chin YS, et al. Prospective study of exercise intervention in prostate cancer patients on androgen deprivation therapy. J Med Imaging Radiat Oncol 2014;58:369-76.

46. Cormie P, Galvão DA, Spry N, et al. Can supervised exercise prevent treatment toxicity in patients with prostate cancer initiating androgen-deprivation therapy: a randomised controlled trial. BJU Int 2015;115:256-66.

47. Buffart LM, Newton RU, Chinapaw MJ, et al. The effect, moderators, and mediators of resistance and aerobic exercise on health-related quality of life in older long-term survivors of prostate cancer. Cancer 2015;121:2821-30.

48. Taaffe DR, Newton RU, Spry N, et al. Effects of different exercise modalities on fatigue in prostate cancer patients undergoing androgen deprivation therapy: a year-long randomised controlled trial. Eur Urol 2017;72:293-9.

49. Vellas B, Guigoz Y, Garry PJ, et al. The Mini Nutritional Assessment (MNA) and its use in grading the nutritional state of elderly patients. Nutrition 1999;15:116-22.

50. Australian Institute of Health and Welfare. Health system expenditure on cancer and other neoplasms in Australia: 2008-09. Cancer series no. 81. Cat. no. 78. Canberra: AlHW, 2013.

51. Klotz L, Vesprini D, Sethukavalan P, et al. Long-term follow-up of a large active surveillance cohort of patients with prostate cancer. $J$ Clin Oncol 2015;33:272-7.

52. Womble PR, Montie JE, Ye Z, et al. Contemporary use of initial active surveillance among men in Michigan with low-risk prostate cancer. Eur Urol 2015;67:44-50.

53. Tosoian JJ, Mamawala M, Epstein JI, et al. Intermediate and longer-term outcomes from a prospective active-surveillance program for favorable-risk prostate cancer. $J$ Clin Oncol 2015;33:3379-85. 